\title{
Research on Key Technologies of Geospatial Information Services Application Based on Grid
}

\author{
Huijuan ZHANG ${ }^{a}$, Li QIAO ${ }^{b}$, Wenqian JIANG ${ }^{c}$, Jinping LI $^{d}$ \\ Institute of Remote Sensing and Digital Earth Chinese Academy of Sciences, Beijing, 100101, \\ China \\ aemail: zhanghj@digitalearth.cn, bemail: qiaol@digitalearth.cn, email: jiangwq@digitalearth.cn, \\ cemail: Lijp@digitalearth.cn
}

Keywords: Geospatial Information; Grid Technology; Parallel and Hidden Search; Collaborative Services

\begin{abstract}
The characteristics of diversity, dynamic, heterogeneity, massive data volume and distribution of geospatial information under internet environment are challenging the traditional geospatial information services. The appearance and development of grid computing provide a reliable environment for the sharing and effective utilization of geospatial information resources. Grid technology is imported into the domain of geospatial information services, this paper studies the geospatial information service system structure based on grid and the key technologies of grid applied in the field of map services, geospatial information search and collaborative service. Main research results are as follows: (1) The design and implementation of grid map service based on GT3.0 are introduced. The architecture of multi grid map services system if put forward. The capability of map service tackling high volume and concurrent access if enhanced by applying multi grid map services system with the policy of Weighted Round-Robin Scheduling.(2) The design of geospatial information search engine based on grid is put forward, the way to build geospatial information parallel and hidden-search platform by grid technology is discussed, and its implementation is also achieved. The efficiency of geospatial information search is promoted by applying geospatial information parallel search technology based on grid.(3) Grid technology is imported into the research of geospatial information collaborative services, the corresponding platform framework design is put forward. The prototype of distributed real-time collaborative mapping system is designed and implemented.
\end{abstract}

\section{Introduction}

The traditional geographic information system is system centered, and different systems are relatively isolated. Network/grid as running platform of geospatial information service, changing the design and application mode of GIS system, is the inheritance and sublimation of GIS. Geospatial information service can provide online personalized geospatial data services, geospatial processing, for the public, businesses and governments, forming a network/grid centered entirely new business service model. With the It becomes Dual drives of technology development and application requirements make it become an inevitable trend from physical geographic information system to intangible geospatial information service. 


\section{Grid Technology}

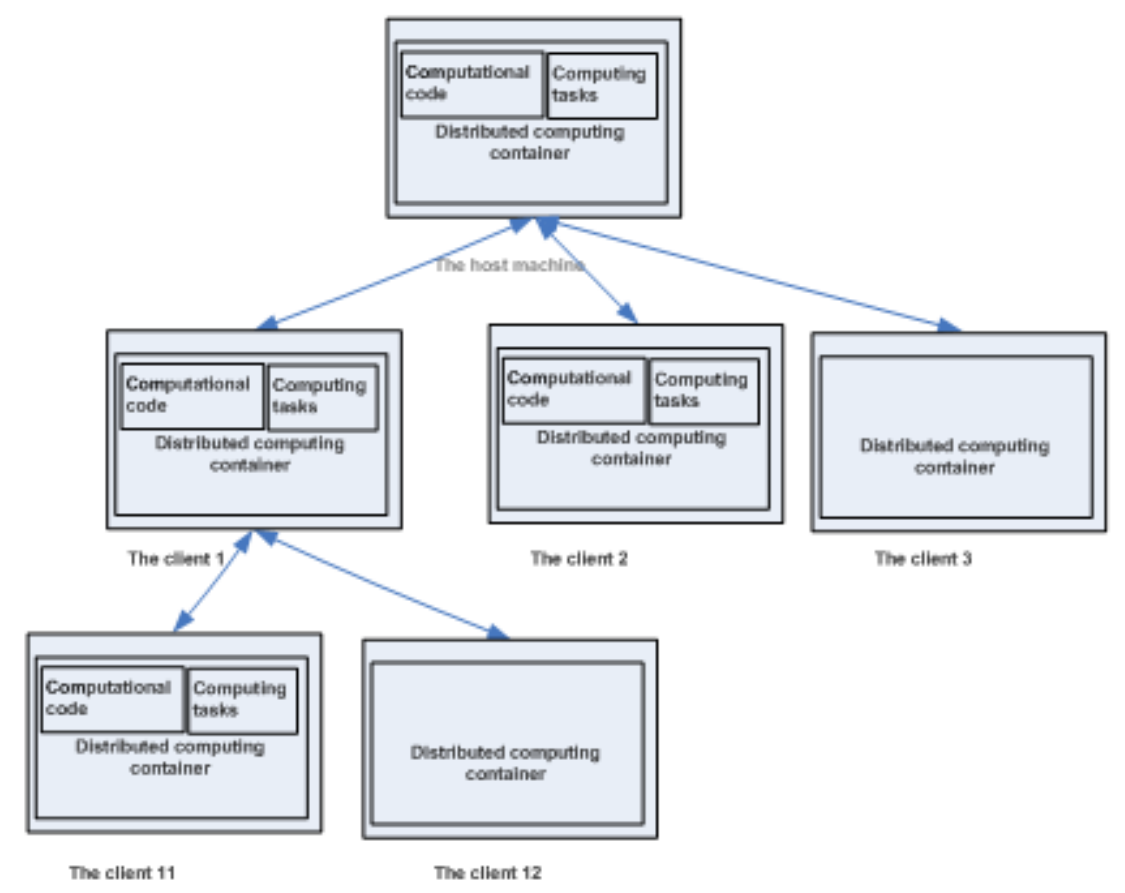

Fig.1. The technology architecture of distributed parallel computing

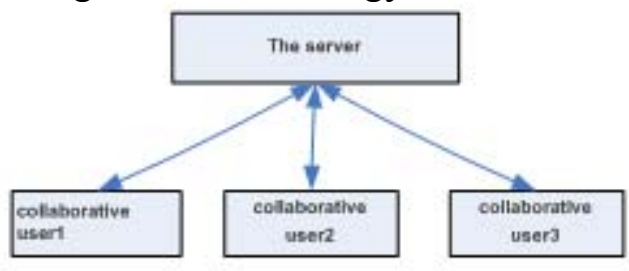

The centralized

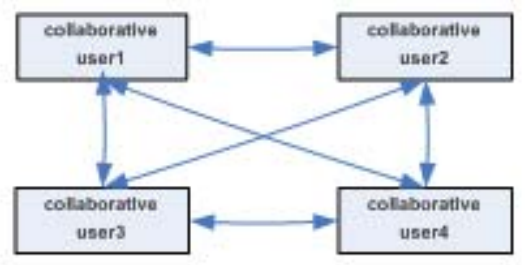

Distrituted

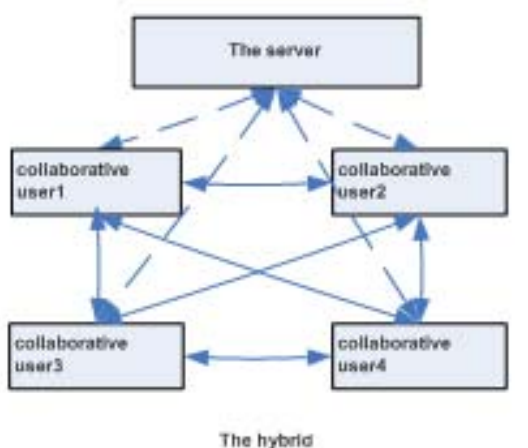

Fig.2. The logic architecture of collaborative computing system

Grid computing develops to be a new calculation model for complex scientific computing along with internet technology, namely is a kind of middleware. Grid computing has two advantages, one is the strong data processing ability, and another can make full use of idle capacity in the network, so as to realize the full sharing of computing resources, storage resources, data resources, information resources, knowledge resources, expert resources [1].

The calculation mode in grid environment mainly includes the following two kinds: the distributed parallel computing and collaborative computing. The technology architecture of distributed parallel computing is as shown in Figure 1. The logic architecture of collaborative computing system is shown in Figure 2.

Distributed computing container is the core of distributed parallel computing, completely responsible for data transmission between the host and client, calculation code management, monitoring and management of the client, and so on, and decomposition and implementation of computing task, as well as combination of computing results initiated by the container. 
In the logic system structure of collaborative computing, it is easy to implement concurrency control of centralized structure, but has a larger traffic, negative network load; the distributed structure has a smaller traffic, but difficult to achieve concurrent control. Hybrid structure organically combines centralized and distributed structure, including the advantages of the two structures [2].

Distributed parallel computing and collaborative computing is two main patterns of grid computing. The application of geospatial information services in constructing distributed parallel and collaborative computing environment, plays an important role in improving computing ability of geospatial information service and group participation.

\section{Map Service Based on Grid}

Grid environment is a Shared, integrated and collaborative environment, combining web map services and grid technology can give full play to the advantages of grid technology, solving some limitations of map service to share resources and collaboration better. Integrating web map service to the grid environment, service oriented grid need to encapsulate map services through grid technology [2].

Taking the Globus Toolkit3.0 as a development tool, grid technology of map service packages map service into grid service and deploys in grid environment. Grid map service in the upper layer of OGSA uses GWSDL (Grid Web services Description Language) to describe map Service functions and releases services;

Integrating and interacting with Web Service technology and other application systems, solves the problems of communication and interoperability of the heterogeneous system, the original map service at a lower level; and using XML technology to exchange information.

The development of grid service is a new programming model, based on GT3.0 tools and Java technology. It allows the application to be abstracted through its interface, and realizes interoperability of cross-platform and cross programming language. Grid map service is realized through inheriting the GridServiceImpl class and realizing the grid service ProtType (GridMapPortType) interface.

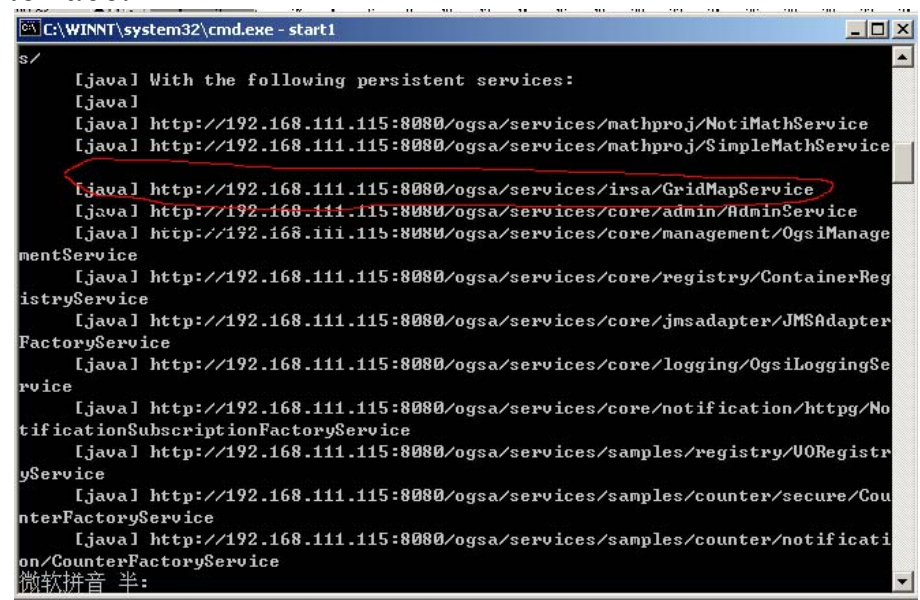

Fig.3. The grid map service of successful deployment

When the deployment file is generated, grid map service can be published to the grid container using the Ant tool, putting its description file, Stub class and implementation class in the corresponding directory. After successful deployment, starting the container, the published grid service can be seen, as shown in Figure 3.

Based on Java technology, a client program is developed to apply grid map service, through calling the operation of getCapabilities to obtain service content, and then initializing.

\section{Geospatial Information Search Service Based on Grid}

The geospatial information search engine based on grid consists of the client, the grid scheduling 
service of geospatial information search and many city geospatial information service subsystems in the distribution of the internet. The design of the space information search engine framework based on grid below in Figure 4.

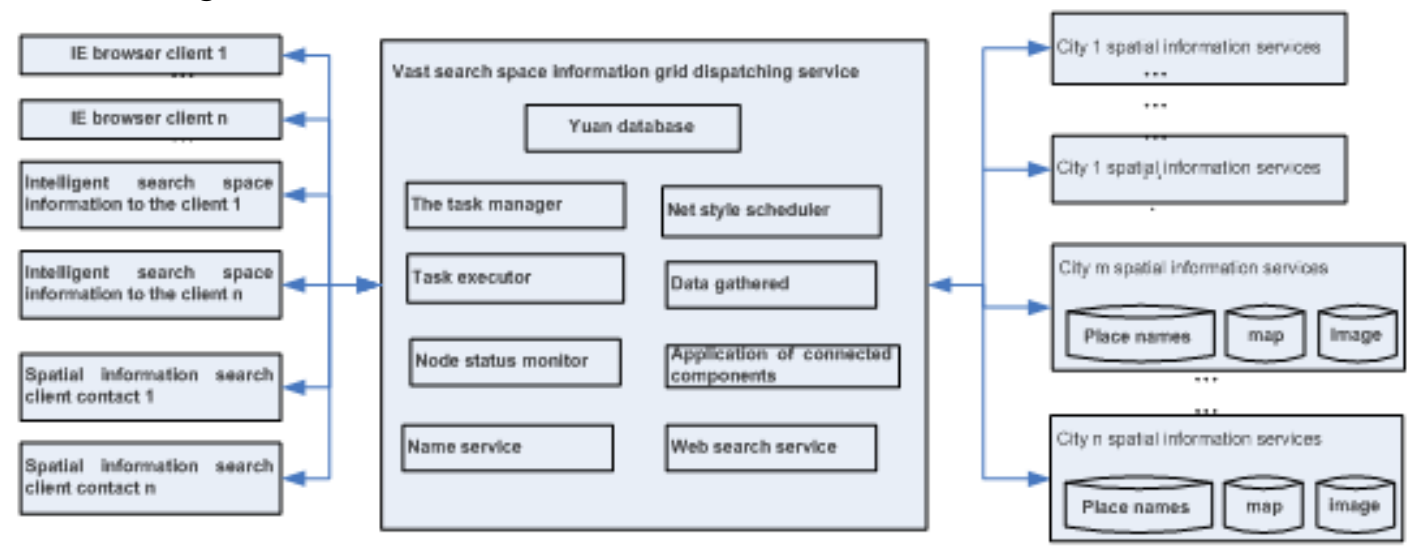

Fig.4. The design of the geospatial information search engine based on grid

The grid scheduling service of geospatial information search is the key to the grid geospatial information search engine service, which provides geospatial information parallel search and contact search service $[3,4]$. The system components designed into Java classes, Grid scheduler, Task executor, Data gathered, Node status monitor are designed to take the initiative to objects, be released Dynamic under the grid network environment. Application of Proactive group communication technology at the same time, realize the parallel processing of space information search and improve the search efficiency [5]. After the completion of the development, the grid scheduling service of geospatial information search needs to be deployed to the server, by calling Proactive. ExposeAsWebService methods published to the Tomcat server.

\section{Geospatial Information Collaborative Service Based on Grid}

Design a kind of geospatial information cooperative service platform based on grid is also important in the face of the requirements of geospatial information service. On the basis of the collaborative platform software, carried out in cooperation with users to geospatial information collaborative browsing, collaborative plotting, collaborative search and so on. The frame of the platform is as shown in Figure 5.

The whole platform consists of the grid client, the grid scheduler, the grid service pool and the geospatial data repository. The grid middleware is the foundation of building a grid environment and the basis for the development and design [4]. On the basis of grid middleware, development of grid service pool, grid scheduler and collaborative information processor. Geospatial data repository is the corresponding resource layer, grid middleware is corresponding to the grid middleware layer, Grid scheduler and collaborative information processor is corresponding to the collaborative support layer, component of GIS and grid service agent is corresponding to the space information service layer, user interface is the corresponding to the application layer. Collaborative services may come from the grid service pool or services provided by the GIS component function. Grid service pool consists of many grid service objects; the grid service object is from different locations of the results of the geospatial information service grid packing. GIS component encapsulates some of vector graphics processing capabilities, to support the collaborative operation for vector graphics data. Using GIS component mode of collaborative services is relatively simple, strong adaptability.

Distributed real-time collaborative cartography is an advanced mapping technology, conforming to the group, distribution and dynamic characteristics of the people working mode in the information age. The different users online and in heterogeneous environment participate in and collaboratively complete the plotting and drawing task. Controlling the implementation of multi-user collaborative operation through grid scheduler, achieves the distributed real-time collaborative mapping in grid environment. 


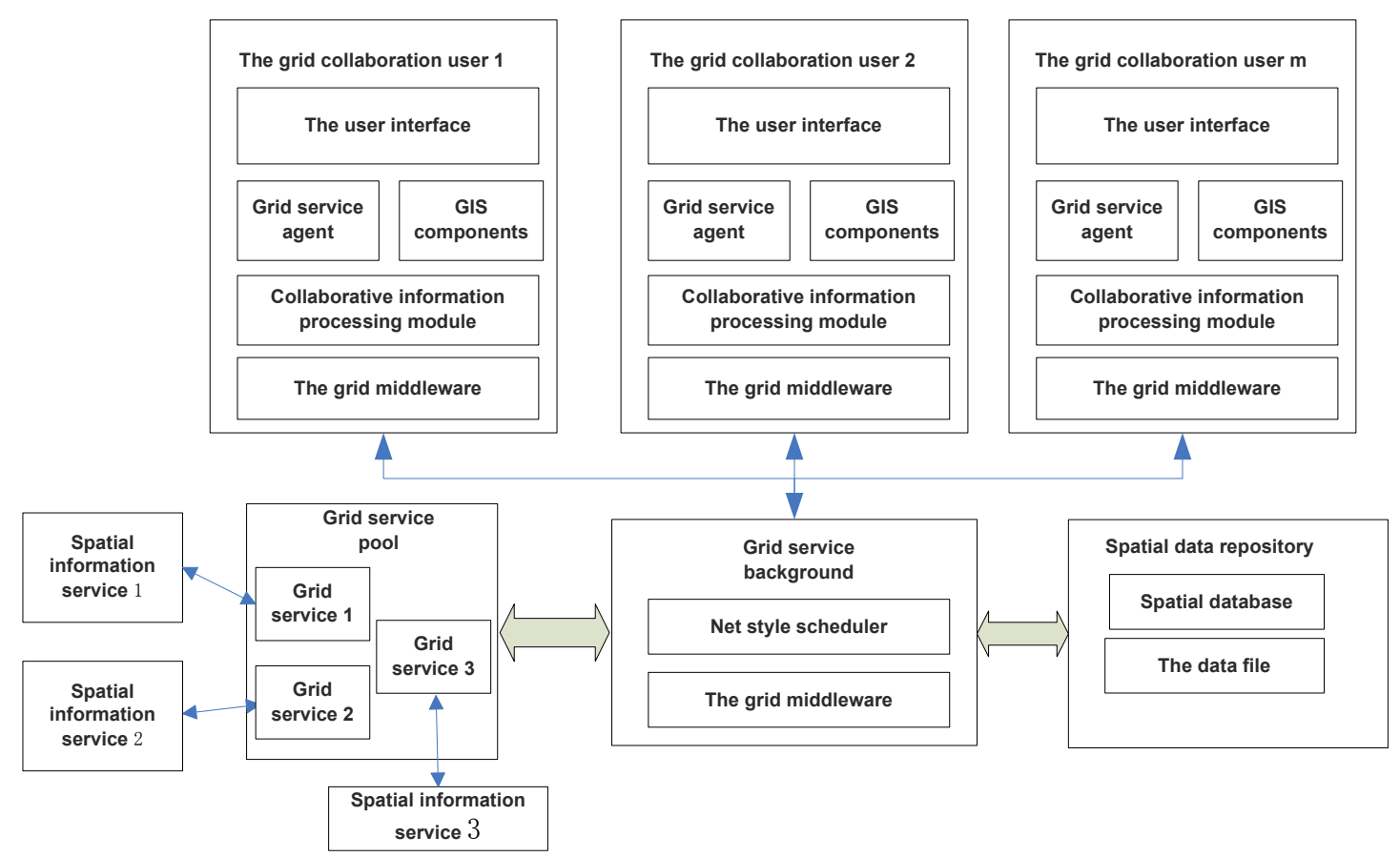

Fig.5. The platform of geospatial information collaborative service based on grid

Using the ProActive Java Library supporting grid development and parallel distributed application under the network environment, builds the grid scheduling services supporting collaborative plotting, and achieves the spotting tasks of multiple staff scattered in different locations participating in map plotting work synchronously, which improves the work efficiency through group cooperation, as shown in Figure 6.

Figure 6 is two operational screenshots in the process of multi-user collaborative editing under the heterogeneous environment, multiple collaborative user to modify the same polygons are simultaneously, the synergy client map interface is consistent, collaborators can see other members in the group activity on the window, mutual synergy can sense.

Design and implementation of distributed real-time collaborative cartography system, you can edit vector graphics file, can zoom graphics in the case of undistorted, enlarge graphics in any area, at the same time provide a real-time communication environment and supporting notation dynamically add and save, collaborative browsing, collaborative editing and coordination meetings and support group decision.
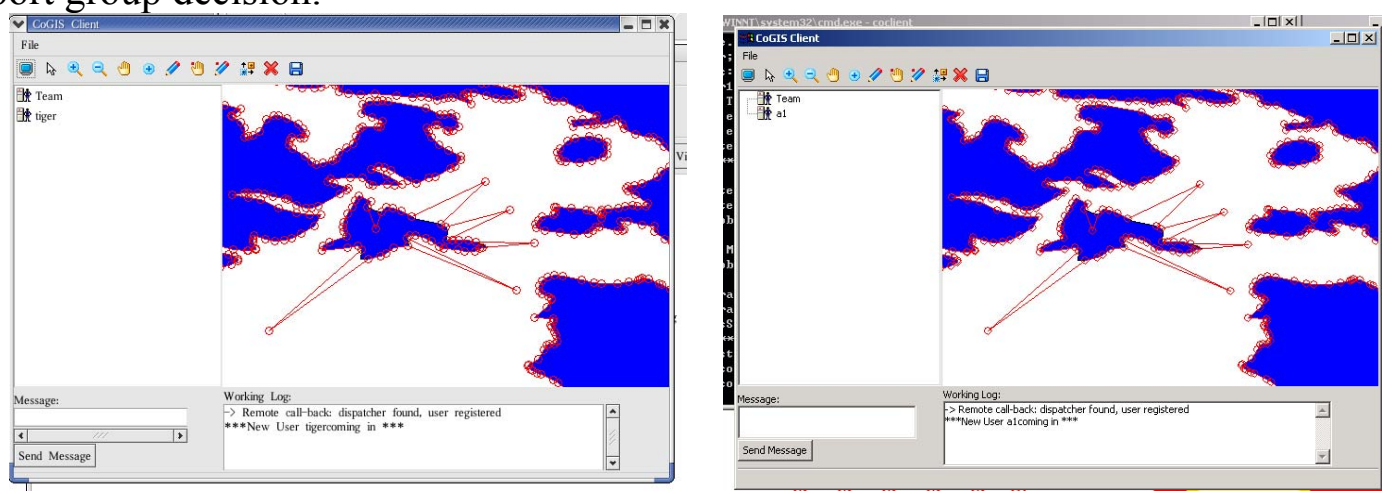

Fig.6. Heterogeneous distributed real-time collaborative mapping scene about Linux (left) and Windows (right).

\section{Conclusion}

(1) Design and implementation of the grid map service based on grid middleware GT3, the multiple grid map service system framework and the method of handling multi-user concurrent requests by take the weighted round-robin scheduling and map subdivision scheduling policy is proposed. 
(2) Geospatial information search engine based on grid was designed, geospatial information grid environment of parallel and contact search is implemented, and thus massive distributed geospatial information search efficiency is improved.

(3) Grid technology into the geospatial information in the study of collaborative services, proposed the corresponding platform framework design, realized the distributed real-time collaborative cartography system prototype.

\section{Acknowledgement}

In this paper, the research was sponsored by the National Science and Technology Support Plan (project No. 2015BAJ08B02) and Industry Database Management Software and Remote Sensing Data Comprehensive Service Platform Construction Project (Project No. 06-Y30B04-9002-13/15).

\section{References}

[1] Xie Zhong, Research on Cooperative Spatial Information Workflow and Its Implementation Technology in Grid GIS [D]. Wuhan, China University of Geosciences, 2012.

[2] Zhang Jianbing, Research on Key Technologies of Geospatial Information Services Based on Grid [D]. Beijing, Institute of Remote Sensing Applications, CAS, 2005.

[3] Meng Xiaoliang,Combination Analysis of Geospatial Information Service Chain in Distributed Environment[D]. Wuhan,Wuhan University, 2009.

[4] BAI Yu-qi, YANG Chong-jun, Research on Spatial Information Search Engine [J]. Journal of China University of Mining 8L Technolog, 2004, 33(1):90-93.

[5] Zhanfeng Shen, Jiancheng Luo, Chenghu Zhou. Architecture design of grid GIS and its applications on image processing based on LAN [J]. Information Sciences, 2004(166):15-17. 